\title{
Clinical outcomes of patients with resected acinar cell carcinoma of the pancreas: A Korea Tumor Registry System Biliary Pancreas (KOTUS-BP) database analysis
}

\author{
Sang Hyun SHIN, In Woong HAN, Jin Seok HEO*
}

Department of Surgery, Samsung Medical Center, Sungkyunkwan University School of Medicine, Seoul, Korea

Introduction: Given the rare incidence of the pancreatic acinar cell carcinoma (PACC), the post-resection clinical outcomes remains still unclear, and treatment strategies have relied on that of pancreatic ductal adenocarcinoma (PDAC).

Methods: The present study retrospectively investigated the clinicopathologic characteristics of resected PACC of 54 patients registered in the Korea Tumor Registry System Biliary Pancreas (KOTUS-BP) database between March 2003 and March 2020.

Results: Among 54 patients, with a mean age of 59 years and a male predominance (81.5\%), there were 38 pure PACC (70.4\%), five ductal differentiations (9.3\%), seven mixed neuroendocrine carcinomas (13.0\%), and four intraductal and papillary variants (7.4\%). A mean tumor size was $4.6 \mathrm{~cm}$ consisting of seven at T1, 25 at T2, and 22 at T3 stages. Metastasis to regional lymph node was identified in 13 patients (24.1\%). Twenty-eight patients (51.9\%) received adjuvant chemotherapy (5-fluorouracil-based [ $\mathrm{n}=15]$; gemcitabine-based $[\mathrm{n}=13])$. The 5 -year survival rate was $54.6 \%$ with a median survival of 78.8 months. In survival comparison according to the stages of American Joint Committee on Cancer system, $\mathrm{N}$ stage (lymph node metastasis) showed significant differences $(p=0.008)$, but T stage did not derive any significant difference.

Conclusions: The resected PACC appears to have clinical outcomes distinct from the PDAC in this nation-wide study. Therefore, large-scale multinational studies are needed to overcome the rarity of PACC and to establish appropriate treatment strategies and staging system. 